\title{
Kann auch ein Vertreter die Wahlleistungs- vereinbarung erfüllen? Wenn der Chefarzt verhindert ist ...
}

Wahlleistungen, wie zum Beispiel eine Chefarztbehandlung, sind für viele Patienten eine interessante Option. Denn gerade in der belastenden Situation einer ernsthaften, unter Umständen sogar lebensbedrohlichen Erkrankung wünscht sich jeder die bestmögliche ärztliche Betreuung. Doch was, wenn der liquidationsberechtigte Arzt einmal aus wichtigen Gründen verhindert ist, seinen Pflichten aus einer Wahlleistungsvereinbarung selbst nachzukommen - wozu er grundsätzlich verpflichtet ist? Unter bestimmten Voraussetzungen kann in solchen Fällen durchaus ein Stellvertreter die vereinbarten Leistungen erbringen, wie es ein aktuelles Urteil des Bundesgerichtshofs jetzt dokumentiert. Beispielsweise darf eine Verhinderung nicht absehbar sein, oder es muss eine individuelle Stellvertretervereinbarung mit dem Patienten bestehen, in der der potenzielle Vertreter namentlich aufgeführt ist. Eine pauschale Verhinderungsklausel in den Allgemeinen Geschäftsbedingungen dagegen reicht nicht aus.

Kann ein Krankenhausarzt, der mit einem Patienten eine Wahlleistungsvereinbarung abgeschlossen hat, die Ausführung seiner Leistung auf einen Vertreter übertragen und dennoch den Honoraranspruch geltend machen? Unter bestimmten Voraussetzungen ist dies durchaus möglich, wie ein aktuelles Urteil des Bundesgerichtshofs vom 20.12.2007 (Aktenzeichen: III ZR 144/07) dokumentiert.

\section{Der aktuelle Streitfall}

Kläger war ein liquidationsberechtiger Chefarzt der Abteilung für Allgemeinchirurgie eines Universitätsklinikums. Eine seiner Patientinnen hatte mit dem Klinikum eine schriftliche, vorgedruckte Wahlleistungsvereinbarung abgeschlossen, die vorsah, dass „im Verhinderungsfall ... die Aufgaben des liquidationsberechtigten Arztes seine Stellvertretung“ übernimmt. Da absehbar war, dass sich der Chefarzt am Tag der geplanten Operation in Urlaub befinden würde, unterzeichnete die Patientin am Vortag noch einen mit handschriftlichen Einträgen versehenen Vordruck, eine „schriftliche Fixierung der Stellvertretervereinbarung“, wonach sie über die Verhinderung des Klägers und den Grund hierfür unterrichtet worden sei. Vermerkt war außerdem, dass die Patientin - da die Verschiebung des Eingriffs medizinisch nicht vertretbar war - darüber belehrt worden sei, dass sie sich entweder wie ein „normaler“ Kassenpatient ohne Zuzahlung von dem jeweils diensthabenden Arzt oder aber zu den Bedingungen des Wahlarztvertrags von dem namentlich bezeichneten Vertreter des Klägers unter Beibehaltung des Liquidationsrechts des Chefarztes operieren lassen könne. In dem Schriftstück war die zweite Alternative angekreuzt. Die vom Chefarzt für die durch den Oberarzt ausgeführte Operation erstellte Rechnung beglich die Patientin jedoch nur zum Teil.

Zunächst wurde die Klage des Chefarztes auf Ausgleich des Restbetrages in zwei Instanzen abgewiesen. Erst der Bundesgerichtshof gab ihm mit folgenden Begründungen Recht.

\section{Kernleistung: in der Regel vom Wahlarzt persönlich}

Grundsätzlich gilt: Der liquidationsberechtigte Arzt muss seine Kernpflichten aus einer Wahlleistungsvereinbarung nach Ansicht des Bundesgerichtshofs selbst erbringen. Dies sei gerade bei einer Chefarztbehandlung der Fall, da der Patient sich damit - gegen die Entrichtung eines zusätzlichen Honorars - die besonderen Erfahrungen und die medizinische Kompetenz des von ihm ausgewählten Arztes sichern wolle.

Eine diesem Grundsatz entsprechende Regelung findet sich auch in der Gebüh- renordnung für Ärzte (GOÄ). Demnach kann ein Arzt Gebühren nur für selbstständig oder unter seiner fachlichen Aufsicht erbrachte ärztliche Leistungen berechnen. Einfache ärztliche und sonstige Verrichtungen darf er delegieren.

\section{Übertragung auf Stellvertreter bei Verhinderung}

Im Falle seiner Verhinderung dürfe ein Wahlarzt jedoch auch die Ausführungen seiner Kernleistungen auf einen Stellvertreter übertragen, so der Bundesgerichtshof, sofern mit dem Patienten eine entsprechende wirksame Vereinbarung bestehe.

In dem konkreten Fall ergebe sich die wirksame Vertretungsregelung allerdings nicht aus der Wahlleistungsvereinbarung selbst, so der Bundesgerichtshof in seiner Urteilsbegründung. Auch wenn dort vorgesehen war, dass im Verhinderungsfall ein Stellvertreter eingesetzt werden könne, sei diese Klausel unwirksam. Da es sich um Allgemeine Geschäftsbedingungen (und keine Individualvereinbarung) handelte, an die sehr strenge Anforderungen gestellt werden, sei ein solcher Passus nur wirksam, wenn er für den Vertragspartner (hier den Patienten) zumutbar sei. Dies sei in diesem Fall jedoch nicht gewährleistet, da die Klausel auch Konstellationen erfasse, in denen die Verhinderung des Wahlarztes bereits zum Zeitpunkt des Abschlusses der Wahlleistungsvereinbarung feststehe. Bereits bei Vertragsabschluss sei hier die von dem Patienten bezweckte Sicherung der Expertise des Wahlarztes für die Heilbehandlung objektiv unmöglich.

Nur namentlich benannter, ständiger Vertreter darf ,einspringen“ Zulässig ist nach Meinung des Bundesgerichtshofs deshalb nur eine Klausel, welche den Einsatz eines Vertreters auf die Fälle beschränkt, in denen die Verhinderung des Arztes zum Zeitpunkt 
des Abschlusses der Wahlleistungsvereinbarung nicht bereits feststeht - etwa weil zum Beispiel eine Krankheit oder ein Urlaub noch nicht abzusehen war oder weil noch nicht bekannt war, dass ein bestimmter Wahlarzt, auf den sich die Wahlleistungsvereinbarung erstreckt, zur Behandlung hinzugezogen werden muss.

Außerdem wird eine Stellvertretervereinbarung nur wirksam, wenn der ständige ärztliche Vertreter namentlich genannt ist. Denn laut der Gebührenordnung ist dieser in gebührenrechtlicher Hinsicht dem Wahlarzt angenähert, weil er kontinuierlich in engem fachlichen Kontakt mit dem liquidationsberechtigten Krankenhausarzt steht. Deshalb kann davon ausgegangen werden, dass er jederzeit voll in die Behandlungsgestaltung des Wahlarztes eingebunden ist. Sein eventueller Einsatz ist daher für den Wahlleistungspatienten weder überraschend noch unzumutbar. Eine weitergehende Vertreterklausel wäre unzumutbar.

\section{Individuelle schriftliche Stell-} vertretervereinbarung möglich

Nach Auffassung des Bundesgerichtshofs reichte im konkreten Fall jedoch die „schriftliche Fixierung einer Stellvertretervereinbarung" als wirksame Vereinbarung aus, um den Chefarzt von seiner persönlichen Verpflichtung, die Operation auszuführen, zu befreien. Damit war der Oberarzt - unter Aufrechterhaltung der Liquidationsbefugnis des Chefarztes - befugt, zu operieren.

Nach Meinung des Gerichts kann sich ein Wahlarzt durch eine individuelle Vereinbarung mit dem Patienten von seiner Pflicht zur persönlichen Leistungserbringung befreien und die Ausführung einem Stellvertreter übertragen. Die Vereinbarung muss aber in jedem Fall schriftlich erfolgen! Außerdem gelten vor Abschluss solcher Stellvertretervereinbarungen besondere Aufklärungspflichten. Denn die Patienten befinden sich in der bedrängenden Sorge um ihre Gesundheit oder gar ihr Überleben. In einer solchen Situation sind sie zu einer ruhigen und sorgfältigen Einschätzung der Lage vielfach jedoch nicht in der Lage.

Zudem fordert der Bundesgerichtshof, den Patienten so früh wie möglich über die Verhinderung des Wahlarztes zu unterrichten und anzubieten, dass ein bestimmter Vertreter die wahlärztli- chen Leistungen zu den vereinbarten Bedingungen erbringe.

\section{Besondere Hinweispflicht beachten}

Sofern die Vereinbarung einer Vertretung im unmittelbaren Zusammenhang mit dem Wahlleistungsvertrag getroffen wird, ist der Patient ausdrücklich auf diese hinzuweisen, meint der Bundesgerichtshof weiter. Dies sei notwendig, da der Patient bei der Aufnahme in das Krankenhaus - einer ohnehin psychisch belastenden Situation - bereits mit der umfangreichen Lektüre der schriftlichen Wahlleistungsvereinbarung und der damit notwendigen Belehrungen befasst ist. Hierdurch besteht eine nicht unerhebliche Gefahr, dass der Patient der Vertretervereinbarung, die letztlich seiner Erwartung widerspricht, nicht die notwendige Aufmerksamkeit zukommen lasse.

\section{Aufklärung über alternative Optionen}

Der Bundesgerichtshof verlangt darüber hinaus, den Patienten über die alternativen Optionen aufzuklären: Er kann auf die wahlärztliche Leistung verzichten und sich ohne Zuzahlung von dem jeweils diensthabenden Arzt behandeln lassen. In diesem Zusammenhang muss man ihn nicht mehr explizit darauf hinweisen, dass er auch in diesem Fall die medizinisch notwendige Versorgung durch hinreichend qualifizierte Ärzte erhält. Nach Auffassung des Gerichts hat dies bereits vor Abschluss der Wahlleistungsvereinbarung stattzufinden. Lässt sich die jeweilige Maßnahme bis zum Ende der Verhinderung des Wahlarztes verschieben, so ist dem Patienten auch diese Möglichkeit zur Wahl zu stellen.

Nicht erforderlich ist es, den Patienten ausdrücklich davon in Kenntnis zu setzen, dass der Wahlarzt auch für die Behandlung durch den Stellvertreter liquidationsberechtigt ist. Sei der Patient über die Option informiert, sich ohne gesondertes Honorar im Rahmen der allgemeinen Krankenhausleistungen behandeln zu lassen und entscheide er sich gleichwohl für die Inanspruchnahme der wahlärztlichen Leistungen zu den vereinbarten Bedingungen durch den Vertreter, müsse ihm klar sein, dass er hierfür auch das für den Wahlarzt anfallende Honorar zahlen müsse.

Außerdem muss der Wahlarzt den Patienten über die genannten Punkte nicht unbedingt selbst aufklären. Der Patient benötige nur die Kenntnis der genannten Tatsachen, um auf einer ausreichenden Grundlage über die Annahme des Angebots auf Abschluss einer Stellvertretervereinbarung zu entscheiden. Auf die besondere Erfahrung und Fachkunde seines Wahlarztes sei er für deren sachgerechte Beurteilung nicht angewiesen.

\section{Trotz Vordruck keine AGB}

Das Gericht musste sich also im konkreten Fall zum einen mit sogenannten Allgemeinen Geschäftsbedingungen (hier die vorformulierte Wahlleistungsvereinbarung) befassen, die einer sehr strengen, gesetzlich vorgeschriebenen, inhaltlichen Kontrolle unterliegen. Zum anderen stellt es inhaltliche Anforderungen für Individualvereinbarungen für Stellvertretung bei Wahlleistungen auf, die mit dem Patienten im Einzelfall ausgehandelt werden (hier die gesonderte „schriftliche Fixierung“).

Obwohl Letztere auch als Vordruck vorlag, wertete der Bundesgerichtshof diese nicht als Allgemeine Geschäftsbedingungen, da die Bedingungen im Einzelnen aufgeführt und ausgehandelt worden seien. Maßgeblich sei dafür nicht, dass die Klinik und der Patient über den Text der Klauseln verhandeln. Vielmehr können auch vorformulierte Vertragsbedingungen ausgehandelt sein, wenn der Verwender (hier die Klinik) sie als eine von mehreren Alternativen anbietet.

Der Vertragspartner (hier der Patient) muss jedoch zum einen über die Auswahlmöglichkeit den Gehalt der Regelung mit gestalten können. Zum anderen darf seine Wahlfreiheit nicht durch eine Einflussnahme des Verwenders sei es nur durch die Gestaltung des Formulare oder in anderer Weise - überlagert werden. Da der Patientin in der „schriftlichen Fixierung“ mehrere Handlungsoptionen zur Wahl standen und keine Beeinflussung des Patienten, sich für eine der Varianten zu entscheiden, zu erkennen ist, seien diese Voraussetzungen im konkreten Fall erfüllt.

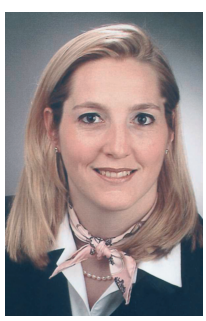

Korrespondenz Dr. iur. Isabel Häser Rechtsanwältin Ehlers, Ehlers \& Partner Widenmayerstraße 29 80538 München i.haeser@eep-law.de 